\title{
Soft Tissue Sarcoma: An Update on Systemic Treatment Options for Patients with Advanced Disease
}

\author{
Patrick Schöffski $^{\mathrm{a}}$ Jasmien Cornillie ${ }^{\mathrm{a}}$ Agnieszka Wozniak $^{\mathrm{a}}$ Haifu Li ${ }^{\mathrm{a}}$ Daphne Hompes ${ }^{\mathrm{b}}$ \\ aDepartment of General Medical Oncology and Laboratory of Experimental Oncology,

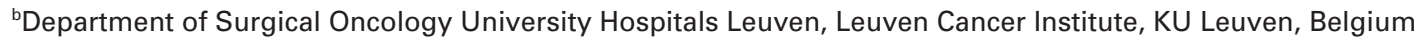

\section{Keywords}

Advanced disease - Chemotherapy - Metastasis .

Soft tissue sarcoma - Targeted agents

\section{Summary}

Sarcomas are a group of rare solid tumours arising from mesenchymal or connective tissue. This review focuses on soft tissue sarcoma and covers general topics such as the epidemiology, age distribution, site of disease, histogenesis, histological subtypes, prognosis and outcome of treatment. In more detail, the article reviews current systemic treatment standards and selected adverse events of agents such as doxorubicin, ifosfamide, trabectedin and pazopanib, and briefly highlights some drugs that are used off-label in specific subtypes of sarcoma.

\section{Introduction}

Sarcomas are a group of rare solid tumours arising from mesenchymal or connective tissue. Collectively, sarcomas account for about $1 \%$ of all adult malignancies. Among the broader family of mesenchymal malignancies, soft tissue sarcomas are the most common tumours, as $80 \%$ of all sarcomas arise from soft tissue, while only $20 \%$ of all sarcomas are bone sarcomas, including osteosarcomas, the Ewing family of tumours and chondrosarcomas. This review focuses on soft tissue sarcoma.

\section{Disease Overview}

\section{Histopathology}

Soft tissue sarcoma consists of a very heterogeneous group of tumours of mesenchymal origin. Of note, not all soft tissue tumours are malignant. The vast majority of mesenchymal lesions are benign entities; they are about a 100 times more frequent than malignant soft tissue sarcoma. The current version of the histopathological classification by the World Health Organization (WHO) defines over 100 different histological variants of benign and malignant soft tissue tumours [1]. The heterogeneity of this disease poses a challenge to the physician caring for patients with sarcoma, as the prognosis and potential response to treatment in a given subtype of a mesenchymal malignancy is difficult to predict.

\section{Incidence and Age Distribution}

In Europe the estimated incidence of soft tissue sarcoma ranges between 2-5/100,000/year [2]. A large number of sarcomas are still misdiagnosed, so the incidence figures available in the literature probably represent an underestimation. The incidence of soft tissue sarcoma increases with higher age but in principle sarcoma does occur in all age groups, and in both female and male patients. The highest incidence is observed between the age of 45 and 90 years. As compared to epithelial tumours, soft tissue sarcoma occurs relatively frequently in children, adolescents and young adults. Soft tissue sarcoma accounts for up to $10 \%$ of all paediatric malignancies and is an important cause of death in the group below 30 years of age. The predominant sarcoma subtype in children below the age of 15 years is rhabdomyosarcoma, which is very uncommon in adult patients. This review focuses on adult soft tissue sarcoma, with exclusion of gastrointestinal stromal tumours.

\section{Primary Site of Disease}

Soft tissue sarcomas can develop at almost any anatomic site, such as the extremities $(60 \%)$, trunk or thorax, abdomen and retroperitoneum, pelvis and the head and neck region. The more common soft tissue sarcomas originate from muscle, nerve tissue, fat or deep skin tissue. For a number of sarcomas the tissue of origin is not well characterised, such as synovial sarcoma.

\section{KARGER \\ Fax +497614520714 \\ Information@Karger.com}

www.karger.com
(C) 2014 S. Karger GmbH, Freiburg

2296-5270/14/0376-0355\$39.50/0

Accessible online at:

www.karger.com/ort
Patrick Schöffski, MD, MPH

Department of General Medical Oncology and Laboratory of Experimental Oncology

Leuven Cancer Institute, University Hospitals Leuven, KU Leuven

Herestraat 49, 3000 Leuven, Belgium

patrick.schoffski@uzleuven.be 


\section{Histogenesis and Histological Subtype}

The relative frequency of the different histological subtypes of soft tissue sarcoma has been described in a number of epidemiological studies, but there is a lot of variation in available published series. The variation can in part be explained by the geographic region in which the epidemiological study was performed. There is also a considerable rate of histological misclassification of sarcomas, which may contribute to the variable distribution in published series. The repeated revisions of the histological classification of sarcomas has also to be taken into consideration. As an example, in the past, malignant fibrous histiocytoma was a very common subtype, but is now virtually non-existent after the more recent revisions of the WHO classification [1]. A large proportion of these tumours are today classified as dedifferentiated liposarcomas, pleomorphic sarcoma or myxofibrosarcoma. Another relevant change in the latest WHO classification is the denotation of haemangiopericytoma as an obsolete term; these tumours are nowadays called solitary fibrous tumours [1]. Another issue making the interpretation of epidemiological data difficult is the fact that some sarcomas, e.g. the superficial skin sarcomas such as dermatofibrosarcoma protuberans or Kaposi sarcomas, are not taken into consideration in many series.

\section{Prognostic Factors and Survival}

The survival of patients with soft tissue sarcoma is far from satisfactory. The disease-free survival has increased substantially over the past few decades due to the introduction of multidisciplinary approaches and with further standardisation of surgery, radiotherapy and the use of systemic therapy. The expected 5-year overall survival (OS) of soft tissue sarcoma across all subtypes and all disease stages, however, remains in the $50 \%$ range according to European and other international series [3]. The prognosis of patients with inoperable, advanced or metastatic soft tissue sarcoma has been static over the past few decades and soft tissue sarcoma remains an unmet medical need.

A number of prognostic factors for survival have been identified, including tumour stage, size, grade, histological subtype, age, presence or absence of metastasis, tumour site and margin of resection. Prognostic nomograms have been developed, helping to predict the survival of patients with newly diagnosed soft tissue sarcoma, but in clinical practice such tools have limited value [4]. The 5-year survival of soft tissue sarcoma is generally dependent on tumour stage. While in early stage disease, 5-year OS rates of approximately $90 \%$ are reached, patients with inoperable, locally advanced or metastatic disease at initial diagnosis have a 5-year survival rate of only $20-50 \%$. Survival also depends on the histological subtype [4]. As an example, malignant peripheral nerve sheath tumours are highly aggressive sarcomas, while liposarcomas appear to exhibit a less aggressive clinical behaviour. However, even between tumours belonging to the same histological subtype there can be a major heterogeneity in terms of tumour aggressiveness. The retrospective interpretation of such data is difficult, as the histopathological classification of sarcomas has changed repeatedly, as described above. Furthermore, a number of sarcomas are still misclassified by pathologists, as these tumours are very rare and the morphological and genetic differentiation of the various subtypes requires expert skills and the availability of specific infrastructure, ranging from electron microscopy to modern molecular diagnostic tools

\section{Outcome of Patients with Local Relapse or}

\section{Distant Metastasis}

Our review focuses on patients with advanced disease. This includes sarcoma patients with local relapse after initial surgery (with or without radiotherapy), patients with distant metastasis and those with both local relapse and metastasis. Patients with local tumour recurrence only have a diseasespecific survival of less than $50 \%$ at 5 years, and those with metastatic relapse have 5-year survival rates in the range of only $15 \%$ [5]. This figure has been disappointingly stable over time. Thus, the major challenge is to achieve improvement of the outcome of patients with such advanced disease states.

\section{Role of Perioperative (Neo-Adjuvant and/or Adjuvant) Treatment}

There have been many attempts to improve the treatment outcome of locally advanced or locally relapsed disease by combining local treatment (surgery, radiotherapy) with systemic therapy, such as neo-adjuvant (preoperative) or adjuvant (postoperative) chemotherapy. There are only limited data supporting the use of such perioperative treatments in sarcoma. Preoperative treatment may be considered in patients for whom the feasibility of a wide oncological resection together with a good functional outcome is questionable [2]. The decision to use neo-adjuvant therapy has to take a number of factors into account, such as tumour histology, patient age, comorbidities and institutional experience. Preoperative chemotherapy does allow assessing the general chemosensitivity of a given disease. If the tumour proves to be radiotherapy or chemotherapy sensitive, neo-adjuvant therapy may render a tumour suitable for conservative rather than radical surgery. If patients with soft tissue sarcoma qualify for preoperative chemotherapy, most oncologists tend to give aggressive combinations. The most commonly used regimen is the combination of doxorubicin and ifosfamide, supported by mesna uroprotection and the use of haematopoietic growth factors. From randomised trials performed in the advanced/ metastatic setting of soft tissue sarcoma, such a combination is known to be associated with a significantly higher rate of objective response induction as compared to single-agent doxorubicin, suggesting that such an approach would also result in the best down-sizing of a tumour in the preoperative setting. Evidence for using more than 2 cytotoxic compounds 
in this setting is lacking, and toxicity usually precludes such an approach [6].

Regional hyperthermia combined with chemotherapy can be considered in the setting of a non-resectable tumour or in patients with regional lymph node metastases $[2,7]$. The role of postoperative or adjuvant chemotherapy remains more controversial. Postoperative systemic therapy can improve disease-free survival, but there is conflicting evidence on improvements in OS from 2 large meta-analyses [8,9]. Adjuvant radiotherapy is frequently used in patients with poor surgical margins or after very complex surgical interventions, mainly in cases with a high risk of local recurrence.

\section{Treatment of Patients with Advanced Disease}

\section{Intent of Treatment}

In inoperable, advanced or metastatic soft tissue sarcoma the therapeutic goals are to achieve control over the disease, to stop or postpone disease progression and to achieve or maintain symptom control for prolonged periods of time. Chemotherapy is the most commonly used treatment for such patients and, although response rates to chemotherapy are very low, about half of all treated patients derive some clinical benefit in advanced stages of the disease [10].

\section{Single-Agent versus Combination Chemotherapy}

If patients have recurrent or progressive soft tissue sarcoma after surgery or extensive synchronous metastasis at initial diagnosis, the most commonly recommended treatment is chemotherapy. The first-line chemotherapy for advanced, metastatic or non-resectable soft tissue sarcoma is typically based on anthracyclines, and the most frequently used compound is doxorubicin [11]. Such chemotherapy should routinely be used as a single agent; only in exceptional circumstances can a combination with a second drug such as ifosfamide be recommended. The majority of clinical studies comparing single agents with combinations failed to show an OS advantage, but have consistently shown improvement in response rates and in progression-free survival (PFS) $[6,12]$. Virtually all of these studies demonstrated that combination chemotherapy is more toxic than single-agent doxorubicin, which is the current standard of care for advanced metastatic soft tissue sarcoma.

While chemotherapy is the mainstay of palliative treatment for locally advanced or metastatic disease, surgery can be considered in selected cases as an adjunct to chemotherapy. A potentially curative approach, albeit efficient only in a small proportion of patients, is secondary resection of metastasis, especially those in the lung [13].

\section{Established Agents}

Doxorubicin and ifosfamide are routinely available and have a very broad label that includes sarcoma in many coun- tries. Trabectedin is approved in Europe and some other countries. Apart from doxorubicin, ifosfamide and trabectedin, a number of other chemotherapeutic drugs are used in selected metastatic soft tissue sarcomas: dacarbazine, gemcitabine, docetaxel and paclitaxel have some value in specific subsets of patients, even though not all of them are officially approved for sarcoma or have been adequately tested for this indication in large randomised trials [12,14-16]. Our report focuses on approved, readily available agents with a proven track record in soft tissue sarcoma, while purposely avoiding reference to systemic therapies with a very low level of supportive evidence.

\section{Doxorubicin}

Doxorubicin is a cytotoxic anthracycline antibiotic that inhibits topoisomerase II, resulting in DNA breakage [17]. The commonly used dose ranges from 60 to $75 \mathrm{mg} / \mathrm{m}^{2}$ once every 3 weeks. The drug is given by intravenous bolus infusion and is the standard of care in this disease. Response rates in clinical trials range between 10 and $25 \%[12,18]$. The dose-limiting toxicity of doxorubicin is cumulative cardiotoxicity, namely a decrease in left ventricular function and other adverse cardiac events. There is also a high incidence of bone marrow depression and stomatitis. Doxorubicin has shown consistent efficacy in a number of sarcoma variants; nevertheless, the results with single-agent doxorubicin are far from being satisfactory. Across the broad range of histological subtypes of soft tissue sarcoma, the OS of patients treated with this drug is still approximately 1 year and the PFS is in the range of only 2-5 months [18]. In the most recent large randomised trial, the response rate to $75 \mathrm{mg} / \mathrm{m}^{2}$ doxorubicin was below $15 \%$ [6]. On the other hand, doxorubicin is easy to administer, can be given on an outpatient basis and patient's acceptance is quite high. Cardiomyopathy is the major limitation. The risk of cardiomyopathy increases with the cumulative dose received. The maximum cumulative dose that should be administered should not extend $550 \mathrm{mg} / \mathrm{m}^{2}$. The risk for cardiac toxicity increases in patients with a history of mediastinal irradiation or concomitant heart disease due to other conditions. Careful monitoring of the left ventricular ejection fraction prior and during treatment is mandatory. According to the American Society of Clinical Oncology guidelines, cardiac monitoring should be done on a regular basis after cumulative doxorubicin doses of $400 \mathrm{mg} / \mathrm{m}^{2}$ and should be repeated after $500 \mathrm{mg} / \mathrm{m}^{2}$ and every additional $50 \mathrm{mg} / \mathrm{m}^{2}$ of doxorubicin. Treatment discontinuation is strongly recommended in patients who develop a decline in left ventricular ejection fraction to below normal limits or in patients who develop clinical congestive heart failure [19].

\section{Ifosfamide}

The second most commonly used drug in soft tissue sarcoma is ifosfamide, which is a cytotoxic alkalising agent belonging to the oxazaphosporine class of compounds. Drugs 
of this family induce DNA cross-links, which blocks tumour cells in late $S$ phase and early G2 phase of the cell cycle. As a single agent after failure of doxorubicin, ifosfamide is usually given at a dose of $8-12 \mathrm{~g} / \mathrm{m}^{2}$ per cycle equally fractioned as single daily doses over 3-5 days. The most commonly used scheme is $3 \mathrm{~g} / \mathrm{m}^{2}$ ifosfamide administered on days 1,2 and 3, repeated every 3 weeks [20]. The drug is infused over $30 \mathrm{~min}$ but can also be given over 4 and $24 \mathrm{~h}$, and has been studied in a number of randomised phase III trials in sarcoma, comparing this drug with other cytotoxic compounds or comparing different ifosfamide schedules to each other. Objective responses to ifosfamide in non-pretreated patients range between $10-25 \%$. In the second-line setting, where the drug is most commonly used, responses are in the range of only $5-8 \%$. The median survival of patients exposed to ifosfamide in second line after doxorubicin failure is in the range of 35-45 weeks with a median time-to-progression of 6-14 weeks [20]. When compared to doxorubicin, ifosfamide achieves very similar results; therefore, ifosfamide is a reasonable alternative if patients cannot be treated with an anthracycline initially. Ifosfamide is associated with a number of potentially severe adverse events including leukopenia, neutropenia, renal toxicity and encephalopathy. The urotoxicity of ifosfamide is related to a metabolite called acrolein, which affects the urinary tract and the bladder and can lead to haemorrhagic cystitis and dysuria. This must be prevented and managed by the concomitant administration of mesna. Another very characteristic adverse event of ifosfamide is encephalopathy, which is seen in up to $10 \%$ of all treated patients. The usual clinical signs are lethargy, hallucinations and other personality changes. This can be treated by an intravenous administration of methylene blue and is fully reversible. A potentially irreversible adverse event is cumulative nephrotoxicity, which can lead to renal insufficiency and even renal failure. This can in part be prevented by aggressive hydration of patients during administration of the anticancer agent.

Apart from being the standard second-line agent in soft tissue sarcoma, ifosfamide is also combined with doxorubicin in clinical situations in which combination chemotherapy is regarded as the better alternative than single-agent treatment [12]. The number of such indications is limited and combination chemotherapy should primarily be used to achieve resectability of locally advanced primary disease or potentially resectable metastases in patients with a good performance status bearing tumour types that are known to be potentially chemotherapy sensitive [21]. Whenever administering doxorubicin in combination with ifosfamide the aim should be to give the highest single-agent doses that can be administered, e.g. $75 \mathrm{mg}$ doxorubicin $/ \mathrm{m}^{2}$ per cycle and approximately 9-10 g ifosfamide $/ \mathrm{m}^{2}$ per cycle with mesna uroprotection and haematopoietic growth factors.

\section{EORTC Trial 62012 Confirms the Current}

\section{Therapeutic Standard}

The most recent clinical trial comparing single-agent doxorubicin to doxorubicin plus ifosfamide in non-pretreated advanced soft tissue sarcoma patients was the European Organisation for Research and Treatment of Cancer (EORTC) 62012. This pivotal trial failed to detect a statistically significant OS benefit but confirmed once again a significant PFS advantage of the combination over single-agent treatment [6]. Response rates were $13.6 \%$ for single-agent doxorubicin versus $26.5 \%$ for the combination. Median PFS increased from 4.6 months for single agent to 7.4 months for the combination. Median OS was 12.8 months for doxorubicin and 14.3 months for doxorubicin/ifosfamide; however, this difference was nonsignificant according to the statistical plan of the trial. The survival at 1 year was $51 \%$ for patients receiving doxorubicin and $60 \%$ for doxorubicin plus ifosfamide [6]. This means that for all clinically relevant endpoints an advantage of combination chemotherapy was demonstrated in this well-powered study, but statistical significance was not reached for the chosen primary endpoint OS. Of note, so far no single randomised phase III trial in metastatic soft tissue sarcoma has been able to generate a clinically relevant and statistically significant OS benefit. The question of whether OS is the ideal endpoint for such trials remains; some experts regard OS as a poor surrogate to assess the efficacy of drugs in such a setting, as survival outcomes are strongly confounded by second- and third-line treatments given to these patients.

\section{Trabectedin}

Trabectedin is an anticancer agent of marine origin acting as a DNA minor-groove binder. The cytotoxic alkaloid was isolated from the Caribbean marine tunicate Ecteinascidia turbinata [22]. The drug is a DNA guanine-specific minor groove binding agent that blocks the cell cycle in late $\mathrm{S}$ and $\mathrm{G}$ phase. Trabectedin is given as a $24-\mathrm{h}$ infusion at a dose of $1.5 \mathrm{mg} / \mathrm{m}^{2}$ every 3 weeks; this can be done during a short hospitalisation or in the outpatient setting through disposable elastomeric pumps [23]. The drug is usually combined with steroids, which have a beneficial effect on the safety profile of this compound. The DNA-repair profile of sarcoma cells predicts the outcome of treatment with trabectedin [24]. Trabectedin is approved in Europe after failure of anthracyclines and ifosfamide in soft tissue sarcoma or in patients who are unsuited to receive these agents. Trabectedin is not approved in the United States for sarcoma. Dose-limiting toxicities include neutropenia and thrombocytopenia but the drug can also cause liver function abnormalities, liver failure, rhabdomyolysis and other severe complications. A critical factor to prevent acute toxicities is to ensure that patients have normal alkaline phosphatase values and normal bilirubin levels, which must be checked prior to each administration of the drug in addition to routine blood counts. Trabectedin shows the best efficacy in the so-called ' $L$ '-sarcomas, i.e. 
leiomyo- and liposarcoma. The results of a small retrospective analysis suggested that trabectedin has clinical activity in synovial sarcoma, even though this tumour type was not included in the trial that led to the registration of this agent in Europe [25]. Within the family of adipocytic tumours, the drug is known to have exquisite activity in the myxoid/round cell liposarcoma subtype. This tumour type is, however, known to be one of the most chemotherapy-sensitive sarcoma subtypes, independent of the drug that is given. No randomised phase III trial for trabectedin has yet been completed. Its approval is based on a randomised phase II study comparing 2 different schedules of administration of the agent, which resulted in a clinical efficacy advantage in terms of time-to-progression for the 24-h 3-weekly schedule over a weekly 3-h infusion schedule [26]. Attempts to establish trabectedin as a first-line standard in non-selected soft tissue sarcomas have failed (EORTC 62091, not yet published).

\section{Other Treatments Including Off-Label Agents}

There is increasing evidence for the off-label use of other drugs in the field of sarcoma, which will not be reviewed in detail here. Gemcitabine with or without docetaxel is commonly used in some specific sarcoma subsets, even though neither of these drugs is approved for this indication. There is considerable doubt whether gemcitabine actually has to be combined with docetaxel. A recent, not yet fully published French metaanalysis of clinical trials indicated that single-agent gemcitabine may be as efficient as the combination while being less toxic. The most relevant trial in this field has compared gemcitabine with and without docetaxel in patients with advanced soft tissue sarcomas [27]. The trial suggested a PFS benefit for the combination and even an advantage in terms of OS. There has been a lot of criticism regarding the Bayesian statistical design of this trial, nevertheless the study forms the basis for the use of this combination in a number of sarcoma settings.

A more recent randomised phase II trial demonstrated superior efficacy of a gemcitabine/dacarbazine combination as compared to dacarbazine alone as second-line therapy. Not only PFS, but also OS was significantly better in the combination treatment group [28]. Of note, 32 of 109 included patients $(29.4 \%)$ in this trial had leiomyosarcoma, which is traditionally known to be responsive to both agents. The OS benefit in this trial is exceptional and warrants further exploration in a more definitive trial. There is also increasing scientific interest in the use of gemcitabine as a single agent or in combination in adjuvant settings as in the case of uterine sarcomas, but at present there is only limited evidence for such a concept. Hence, such treatments should not be given outside clinical studies. Another drug that is frequently used in the field of sarcoma is paclitaxel, which shows exquisite activity in smaller series of patients with advanced vascular tumours, especially angiosarcomas. Paclitaxel has not been studied in large-scale, randomised, multi-centre trials, so the evidence level is still low [16].
Adverse Events Observed with Common Cytotoxic Agents

\section{for Treatment of Sarcoma}

Every cytotoxic compound used for the treatment of soft tissue sarcoma has specific adverse events. All these drugs are relatively non-specific and interact not only with the malignant cells but also with normal tissue, especially in cells with a high proliferation rate. Doxorubicin is associated with cardiomyopathy, ifosfamide with uro- and nephrotoxicity and encephalopathy [29]. Trabectedin can cause haematological events, gastrointestinal side effects and rhabdomyolysis [30]. Gemcitabine is associated with myelosuppression, docetaxel with dose-dependent sensory neuropathy and oedema, and paclitaxel with haematological events, neurotoxicity and arthralgia, just to mention a few typical events.

Chemotherapy with any of these agents can have a severe negative impact on the quality of life of the patients during the actual treatment period, while the quantity, quality and duration of responses achieved are very unsatisfactory in the majority of cases. Whenever considering chemotherapy in the palliative setting of metastatic soft tissue sarcoma, quality of life and other patient-related factors have to be taken into account before making definitive decisions. The key problem in soft tissue sarcoma remains primary resistance to chemotherapy as illustrated by the low response rates and the rapid occurrence of secondary resistance during or after initial treatment in the vast majority of patients. The results of second- and third-line chemotherapies in sarcoma are usually much worse than the outcome of first-line treatment with an anthracycline, but in exceptional cases such salvage treatments can result in surprising patient benefit and should always be considered, at least in patients with good performance status and good organ function. Most patients with advanced or metastatic soft tissue sarcoma receive more than 1 line of palliative treatment. Only a very small percentage of patients achieve long-term disease control with systemic therapy only, but it does occur.

\section{New Cytotoxic Agents in Late Stage \\ of Clinical Development}

A number of innovative agents with promising anti-tumour activity in soft tissue sarcoma in earlier studies are currently being tested in international randomised phase III trials. These include drugs of the oxazaphosphorine family such as palifosfamide, a DNA-alkylating metabolite of ifosfamide, and TH-302, a hypoxia-activated prodrug selectively targeting hypoxic regions of solid tumours [31,32]. Both agents have been developed for the first-line setting of metastatic soft tissue sarcoma. Results of the randomised, double-blind, placebo-controlled phase III trial comparing doxorubicin plus palifosfamide versus doxorubicin alone as first-line treatment for advanced soft tissue sarcoma were presented at the European Cancer Organisation (ECCO) congress in September 2013. Combination treatment demonstrated only a 3.1-week improvement in PFS and this difference was statistically non- 
significant [33]. As a consequence, development of the compound for this indication was terminated. Recently, the phase III trial comparing doxorubicin with doxorubicin/TH-302 completed accrual; results are pending. In addition, eribulin mesylate, a synthetic analogue of halichondrin $\mathrm{B}$, a compound originally isolated from marine sponges, is currently being compared to dacarbazine in a pivotal trial in previously treated L-sarcomas based on the promising results of a previous EORTC phase II study [34]. Accrual for this trial has been completed and first results are expected in 2014.

\section{Pazopanib as Prototype Targeted Agent \\ in Soft Tissue Sarcoma}

Pazopanib is the first non-chemotherapeutic anticancer agent approved by regulatory authorities for soft tissue sarcoma. Pazopanib interferes with the vascular endothelial growth factor and platelet-derived growth factor pathways. The approval of this oral anti-angiogenic agent is based on the EORTC trial 62072 (PALETTE). Pazopanib is an orally bioavailable tyrosine kinase inhibitor (TKI), and showed evidence of efficacy in mesenchymal diseases already during a dose-finding phase I study and in a phase II study performed by the EORTC (trial 62043) [35,36]. The latter study revealed promising PFS rates. In the following pivotal multi-national phase III trial EORTC 62072, pazopanib was compared at the standard oral dose of $800 \mathrm{mg}$ daily to a matching placebo [37]. This study confirmed the efficacy of the angiogenesis inhibitor in heavily pretreated patients with soft tissue sarcoma previously exposed to systemic cytotoxic therapy. The median PFS in patients treated with pazopanib was 20 weeks as compared to 7 weeks with placebo, which was statistically significant. The benefit of pazopanib was independent of the number of prior lines of systemic therapy, the performance status at baseline or the type of soft tissue sarcoma. Overall survival curves suggested a benefit of pazopanib over placebo (median OS 12.6 versus 10.7 months), but this difference was not statistically significant. The OS analysis was confounded by various factors, including imbalanced post-study treatments. Of the placebo-treated group in the registration trial, $75 \%$ received further treatments and the anticancer follow-up therapy included chemotherapy, targeted therapies, radiotherapy, surgery or other interventions [36]. Pazopanib is a drug that mainly induces disease stabilisation: partial responses are rare (4\%), but stable disease is achieved in about half of all patients. In Europe, pazopanib is approved for the treatment of patients with advanced soft tissue sarcoma, except for patients with the histological subtypes gastrointestinal stromal tumour and adipocytic sarcoma who have received prior chemotherapy or for patients who are unsuited for such therapy.

\section{Guidelines}

A number of academic organisations have published guidelines for the treatment of inoperable, advanced, metastatic sarcoma. The European Society for Medical Oncology
(ESMO) guidelines recommend as first-line treatment anthracyclines as single agent or in combination with ifosfamide or single-agent ifosfamide if there are specific contra-indications [38]. Second-line treatments include ifosfamide at standard doses if patients have not been previously treated with this agent during first-line treatment. A high-dose ifosfamide schedule is recommended by ESMO if the drug had been previously used at a lower dose. Trabectedin is recommended especially for L-sarcomas. Best supportive care is considered a reasonable option for a number of patients instead of continued chemotherapy.

The British Sarcoma Group recommends single-agent doxorubicin or ifosfamide or doxorubicin and ifosfamide in the first-line setting [11]. These guidelines were published before the results of EORTC 62012 (showing the failure of the combination of doxorubicin plus ifosfamide to generate an OS benefit) had been presented. The British Sarcoma Group recommends second-line treatments with either ifosfamide, trabectedin, gemcitabine and docetaxel or the older drug dacarbazine [11].

The National Comprehensive Cancer Network (NCCN) has published more detailed North American guidelines, and the list of drugs considered for treatment of sarcoma reflects the very common practice of off-label use of anticancer drugs in the United States, which may not be applicable to the European situation. Many NCCN recommendations for treatment of subtypes of soft tissue sarcoma are not based on high-level scientific evidence and the guidelines are not in line with drug approval and reimbursement criteria in most countries.

\section{Conclusions}

Soft tissue sarcoma is a very heterogeneous and rare family of malignancies that can occur in all age groups and all anatomical sites. Treatment outcomes are far from being satisfactory, especially in the subset of patients with inoperable, locally advanced and/or metastatic disease. The diagnosis of soft tissue sarcoma poses a significant diagnostic and therapeutic challenge to all involved disciplines. The classification of soft tissue sarcoma is undergoing continuous evolution, and a number of subtypes can only be diagnosed correctly when sophisticated molecular diagnostic tests are applied and results are interpreted by expert geneticists and pathologists. Treatment planning requires close interaction between all involved disciplines represented within an expert sarcoma team that meets on a regular basis and updates institutional treatment standards based on the continuously increasing scientific evidence in this field.

To achieve optimal treatment results, patients with presumed, newly diagnosed or progressive soft tissue sarcoma should be transferred to reference sites. These reference institutions should have a track record of multidisciplinary sarcoma care and ongoing surgical, radiotherapeutic and/or 
chemotherapy trials in soft tissue sarcoma [38]. Ideally, such institutions should be embedded in multi-national sarcoma research initiatives and should have strong links with patient advocacy groups with a specific interest in sarcoma. Reference sites should also have the infrastructure for performing basic or translational research in the field of sarcoma and have access to comprehensive diagnostic tools including, but not limited to, imaging (computed tomography, magnetic resonance imaging, $\left[{ }^{18} \mathrm{~F}\right]$ fluorodeoxyglucose-positron emission tomography), histopathology (conventional staining, immunohistochemistry, electron microscopy) and genetic testing (cytogenetics, fluorescence in situ hybridisation, array comparative genomic hybridisation, and -omics).

Furthermore, the heterogeneity across the histological subtypes, also in terms of chemosensitivity, emphasises the importance of stratification by histological subtypes or inclusion of patients with a single histological subtype in future prospective randomised trials in soft tissue sarcoma.

\section{Disclosure Statement}

The Department of General Medical Oncology has received funding for participation in multiple sarcoma trials led by different academic groups and pharmaceutical companies. P. Schöffski, MD, MPH has received honoraria from pharmaceutical companies (Pfizer, PharmaMar, Glaxo Smith Kline) for participation in advisory and educational events related to the treatment of soft tissue sarcoma. P. Schöffski, MD, MPH, J. Cornillie, MD, H. Li, MD, and A. Wozniak, PhD have received generous support from Fonds Wetenschappelijk Onderzoek - Vlaanderen (FWO Grants GA01311N, G081611N) and from Stichting tegen Kanker for basic and translational research in the field of soft tissue sarcoma. This article is an updated version of a previously published review article (Belg J Med Oncol 2013;7:80-88). Written permission from the publisher was obtained.

\section{References}

1 Fletcher CDM, et al. (eds): WHO classification of tumours of soft tissue and bone, 4th ed. Lyon, IARC Press, 2013

-2 Casali PG, Blay JY; ESMO/CONTICANET/ EUROBONET consensus panel of experts: Soft tissue sarcomas: ESMO Clinical Practice Guidelines for diagnosis, treatment and follow-up. Ann Oncol 2010;21 Suppl 5:v198-203.

-3 Stiller CA, Trama A, Serraino D, et al.: Descriptive epidemiology of sarcomas in Europe: Report from the RARECARE project. Eur J Cancer 2013;49: 684-695.

4 Eilber FC, Brennan MF, Eilber FR, et al.: Validation of the postoperative nomogram for 12-year sarcoma-specific mortality. Cancer 2004;101:22702275.

5 Zagars GK, Ballo MT, Pisters PW, et al. Prognostic factors for disease-specific survival after first relapse of soft-tissue sarcoma: Analysis of 402 patients with disease relapse after initial conservative surgery and radiotherapy. Int J Radiat Oncol Biol Phys 2003;57:739-747.

6 Judson I, Verweij J, Gelderblom H, et al.: Doxorubicin alone versus intensified doxorubicin plus ifosfamide for first-line treatment of advanced or metastatic soft-tissue sarcoma: A randomised controlled phase 3 trial. Lancet Oncol 2014; 15:415-423.

7 Issels RD, Lindner LH, Verweij J, et al.: Neo-adjuvant chemotherapy alone or with regional hyperthermia for localised high-risk soft-tissue sarcoma: A randomised phase 3 multicentre study. Lancet Oncol 2010;11:561-570.

8 Tierney JF, Mosseri V, Stewart LA, et al.: Adjuvant chemotherapy for soft-tissue sarcoma: Review and meta-analysis of the published results of randomised clinical trials. Br J Cancer 1995;72:469-475.

$\checkmark$ Pervaiz N, Colterjohn N, Farrokhyar F, et al.: A systematic meta-analysis of randomized controlled trials of adjuvant chemotherapy for localized resectable soft-tissue sarcoma. Cancer 2008;13:573581.

10 Karavasilis V, Seddon BM, Ashley S, et al.: Significant clinical benefit of first-line palliative chemotherapy in advanced soft-tissue sarcoma: retro- spective analysis and identification of prognostic factors in 488 patients. Cancer 2008;112:1585-1591.

11 Grimer R, Judson I, Peake D, Seddon B: Guidelines for the management of soft tissue sarcomas. Sarcoma 2010;2010:506182.

12 Santoro A, Tursz T, Mouridsen H, et al.: Doxorubicin versus CYVADIC versus doxorubicin plus ifosfamide in first-line treatment of advanced soft tissue sarcomas: A randomized study of the European Organization for Research and Treatment of Cancer Soft Tissue and Bone Sarcoma Group. J Clin Oncol 1995;13:1537-1545.

13 Kim S, Ott HC, Wright CD, et al.: Pulmonary resection of metastatic sarcoma: Prognostic factors associated with improved outcomes. Ann Thorac Surg 2011;92:1780-1786.

14 Pautier P, Floquet A, Penel N, et al.: Randomized multicenter and stratified phase II study of gemcitabine alone versus gemcitabine and docetaxel in patients with metastatic or relapsed leiomyosarcomas: A Federation Nationale des Centres de Lutte Contre le Cancer (FNCLCC) French Sarcoma Group Study (TAXOGEM study). Oncologist 2012;17:1213-1220.

15 Bramwell V, Blackstein M, Belanger K, et al.: A phase II study of docetaxel in chemotherapynaïve patients with recurrent or metastatic adult soft tissue sarcoma. Sarcoma 1998;2:29-33.

16 Schlemmer M, Reichardt P, Verweij J, et al.: Paclitaxel in patients with advanced angiosarcomas of soft tissue: A retrospective study of the EORTC soft tissue and bone sarcoma group. Eur J Cancer 2008;44:2433-2436.

17 Riggs CE: Antitumor antibiotics; in Perry MC (ed.): The chemotherapy sourcebook. Lippincott Williams and Wilkins, 2001, pp. 231-234.

18 Lorigan P, Verweij J, Papai Z, et al.: Phase III trial of two investigational schedules of ifosfamide compared with standard-dose doxorubicin in advanced or metastatic soft tissue sarcoma: A European Organisation for Research and Treatment of Cancer Soft Tissue and Bone Sarcoma Group Study. J Clin Oncol 2007;25:3144-3150.

19 Hensley M, Hagerty K, Kewalramani T, et al.: American Society of Clinical Oncology 2008 clini- cal practice guideline update: Use of chemotherapy and radiation therapy protectants. J Clin Oncol 2008;27:127-145.

20 Van Oosterom AT, Mouridsen HT, Nielsen OS, et al.: Results of randomised studies of the EORTC Soft Tissue and Bone Sarcoma Group (STBSG) with two different ifosfamide regimens in first- and second-line chemotherapy in advanced soft tissue sarcoma patients. Eur J Cancer 2002;38:2397-2406.

21 Blay JY, Sleijfer S, Schöffski P, et al.: Internationa expert opinion on patient-tailored management of soft tissue sarcomas. Eur J Cancer 2014;50:679-689.

22 Schöffski P, Dumez H, Wolter P, et al.: Clinical impact of trabectedin (ecteinascidin-743) in advanced/metastatic soft tissue sarcoma. Expert Opin Pharmacother 2008;9:1609-1618.

23 Schöffski P, Cerbone L, Wolter P, et al.: Administration of 24-h intravenous infusions of trabectedin in ambulatory patients with mesenchymal tumors via disposable elastomeric pumps: An effective and patient-friendly palliative treatment option. Onkologie 2012;35:14-17.

24 Schöffski P, Taron M, Jimeno J, et al.: Predictive impact of DNA repair functionality on clinical outcome of advanced sarcoma patients treated with trabectedin: A retrospective multicentric study. Eur J Cancer. 2011;47:1006-1012.

25 Dileo O, Sanfilippo R, Grosso F, et al.: Trabectedin (T) in advanced, pretreated synovial sarcomas (SS): A retrospective analysis of 39 patients (pts) from three European institutions. 2010 ASCO Annual Meeting Abstracts. J Clin Oncol 2010;28: No. 7S (abstract 10030).

26 Demetri GD, Chawla SP, Von Mehren M, et al.: Efficacy and safety of trabectedin in patients with advanced or metastatic liposarcoma or leiomyosarcoma after failure of prior anthracyclines and ifosfamide: Results of a randomized phase II study of two different schedules. J Clin Oncol 2009;27: 4188-4196.

27 Maki RG, Wathen JK, Patel SR, et al.: Randomized phase II study of gemcitabine and docetaxel compared with gemcitabine alone in patients with metastatic soft tissue sarcomas: Results of sarcoma alliance for research through collaboration study 
002. J Clin Oncol 2007;25:2755-2763. Erratum in: J Clin Oncol 2007;25:3790.

28 Garcia-del-Muro X, López-Pousa A, Maurel J, et al.: Randomized phase II study comparing gemcitabine plus dacarbazine alone in patients with previously treated soft tissue sarcoma: A Spanish Group for Research on Sarcomas Study. J Clin Oncol 2011;29:2528-2533.

29 Grochow LB: Covalent DNA-binding drugs; in Perry MC (ed.): The chemotherapy sourcebook. Lippincott Williams and Wilkins, 2001, pp. 196-197.

30 Schöffski P, Wolter P, Clement P, et al.: Trabectedin (ET-743): Evaluation of its use in advanced softtissue sarcoma. Future Oncol 2007;3:381-392.

31 Jones B, Komarnitsky P, Miller GT, et al.: Anticancer activity of stabilized palifosfamide in vivo: Schedule effects, oral bioavailability, and enhanced activity with docetaxel and doxorubicin. Anticancer Drugs 2012;23:173-184.
32 Liu Q, Sun JD, Wang J, Ahluwalia D, et al.: TH-302, a hypoxia-activated prodrug with broad in vivo preclinical combination therapy efficacy: Optimization of dosing regimens and schedules. Cancer Chemother Pharmacol 2012;69:1487-1498.

33 Ryan CW, Schöffski P, Merimsky O, et al.: A phase 3 multicenter, international, randomized, doubleblind, placebo-controlled study of doxorubicin plus palifosfamide vs. doxorubicin plus placebo in patients with front-line metastatic soft tissue sarcoma. ECCO Congress 2013, Amsterdam.

34 Schöffski P, Ray-Coquard IL, Cioffi A, et al.: Activity of eribulin mesylate in patients with soft-tissue sarcoma: A phase 2 study in four independent histological subtypes. Lancet Oncol 2011;12:10451052.

35 Hurwitz HI, Dowlati A, Saini S, et al.: Phase I trial of pazopanib in patients with advanced cancer. Clin Cancer Res 2009;15:4220-4227.
6 Sleijfer S, Ray-Coquard I, Papai Z, et al.: Pazopanib, a multikinase angiogenesis inhibitor, in patients with relapsed or refractory advanced soft tissue sarcoma: A phase II study from the European Organisation for Research and Treatment of Cancer Soft Tissue and Bone Sarcoma Group (EORTC study 62043). J Clin Oncol 2009;27:31263132.

37 Van der Graaf WT, Blay JY, Chawla SP, et al.: Pazopanib for metastatic soft-tissue sarcoma (PALETTE): A randomised, double-blind, placebo-controlled phase 3 trial. Lancet 2012;379: 1879-1886.

38 The ESMO/European Sarcoma Network Working Group. Soft tissue and visceral sarcomas: ESMO Clinical Practice Guidelines for diagnosis, treatment and follow-up. Ann Oncol 2012;23 (Suppl 7):vii92-99. 